\title{
ON NORMAL AND STRONGLY NORMAL LATTICES
}

\section{EL-BACHIR YALLAOUI}

Department of Mathematics

Polytechnic University

Brooklyn, NY 11201

(Received June 12, 1991 and in revised form October 21, 1991)

\begin{abstract}
In this paper we will investigate the properties of normality and strong normality of lattices and their relationships to zero-one measures. We will eventually establish necessary and sufficient conditions for lattices to be strongly normal. These properties are then investigated in the case of separated lattices.
\end{abstract}

KEY WORDS AND PHRASES. Normal, strongly normal, prime complete and Lindelöf lattices, Filters, prime filters and ultrafilters.

1991 AMS SUBJECT CLASSIFICATION CODE. $28 \mathrm{C} 15$.

\section{INTRODUCTION.}

Let $X$ be an arbitrary set and $\ell$ a lattice of subsets of $X . \ell(\ell)$ is the algebra generated by $\ell$, and $I(\ell)$ denotes the non-trivial zero-one valued finitely additive measures on $\ell(\ell) . I_{R}(\ell)$ will denote those $\mu \in I(\ell)$ that are $\ell$-regular, and $I_{R}^{\sigma}(\ell)$ consists of those $\mu \in I_{R}(\ell)$ which are countably additive.

We first consider a number of equivalent characterizations of $l$ being a normal lattice, and then introduce the concept of a strongly normal lattice and give an alternate characterization of a lattice being strongly normal.

We associate next with $\ell$, a lattice $W_{\sigma}(\ell)$ in $I_{R}^{\sigma}(\ell)$. Assuming $\ell$ is disjunctive, $W_{\sigma}(\ell)$ is always a replete lattice. We give necessary and sufficient conditions for $W_{\sigma}(\ell)$ to be a prime complete lattice. Next, we consider the set $I_{R}^{\circ}(\ell)$ with the topology of closed sets given by $\tau W_{\sigma}(\ell)$ consisting of arbitrary intersections of sets of $W_{\sigma}(\ell)$. We investigate this topological space to some extent giving necessary and sufficient conditions for it to be $T_{2}$; similarly we given necessary and sufficient conditions for it to be Lindelöf; finally we consider conditions when it is normal.

The notations and terminology used in this paper are standard and are consistent with [1], [2], [5], [6] and [7]. Our work on normal lattices is closely related to work done in [3] and [4].

We begin with a brief review of some notations and some definitions for the reader's convenience.

2. DEFINITIONS AND NOTATIONS.

Let $X$ be an abstract set and $\ell$ a lattice of subsets of $X$. We will always assume that $X$ are in $\mathcal{L}$. If $A \subset X$ then we will denote the complement of $A$ by $A^{\prime}$ i.e., $A^{\prime}=X-A$. If $\mathcal{L}$ is a lattice of subset of $X$ then $\ell^{\prime}$ is defined $\ell^{\prime}=\left\{L^{\prime} \mid L \in \ell\right\}$

\section{LATTICE TERMINOLOGY}

DEFINITION 2.1. Let $\ell$ be a Lattice of subsets of $X$. We say that $\ell$ is:

1) $\delta$-lattice if it is closed under countable intersections.

2) Separating or $T_{1}$ if $x, y \in X ; x \neq y$ then $\exists L \in \mathcal{L}$ such that $x \in L$ and $y \notin L$. 
3) Hausdorff or $T_{2}$ if $x, y \in X ; x \neq y$ then $\exists A, B \in \mathcal{L}$ such that $x \in A^{\prime}, y \in B^{\prime}$ and $A^{\prime} \cap B^{\prime}=0$.

4) Disjunctive if for $x \in X$ and $L \in \mathcal{l}$ where $x \notin L, \exists A \in \mathcal{l}$ such that $x \in A$ and $A \cap L=0$.

5) $\mathcal{L}$ is normal if for $A, B \in \mathcal{L}$ where $A \cap B=\theta, \exists \widetilde{A}, \widetilde{B} \in \mathcal{L}$ such that $A \subset \widetilde{A}^{\prime}, B \subset \widetilde{B}^{\prime}$ and $\widetilde{A}^{\prime} \cap \widetilde{B}^{\prime}=\theta$.

6) $\ell$ is compact if any covering of $X$ by $\ell$ sets has a finite subcovering.

7) $\mathcal{L}$ is countably compact if any countable covering of $X$ by $\mathcal{L}^{\prime}$ sets has a finite subcovering.

8) $\ell$ is Lindelöf if any covering of $X$ by $\ell^{\prime}$ sets has a countable subcovering

$\ell(\ell)=$ the algebra generated by $\ell$.

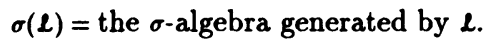

$\delta(\ell)=$ the lattice of countable intersections of sets of $\ell$.

$\tau(\ell)=$ the lattice of arbitrary intersections of sets of $\ell$.

\section{MEASURE TERMINOLOGY}

Let $\ell$ be a lattice of subsets of $X . M(\ell)$ will denote the set of finite valued bounded finitely additive measures on $\mathcal{l}(\ell)$. Clearly since any measure in $M(\ell)$ can be written as a difference of two non-negative measures there is no loss of generality in assuming that the measures are non-negative, and we will assume so throughout this paper. We will say that a measure $\mu$ of $M(\ell)$ is regular if for

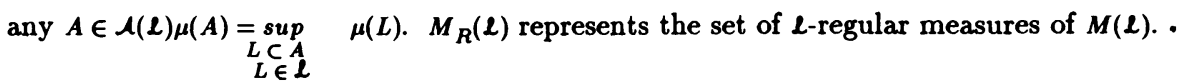

\section{DEFINITION 2.2 .}

1) A measure $\mu \in M(\ell)$ is said to be $\sigma$-smooth on $\ell$, if for $L_{n} \in \mathcal{L}$ and $L_{n} \downarrow \ell$; then $\mu\left(L_{n}\right) \rightarrow 0$.

2) A measure $\mu \in M(\ell)$ is said to be $\sigma$-smooth on $\mathcal{\Lambda}(\ell)$, if for $A_{n} \in \mathcal{\Lambda}(\ell), A_{n} \downarrow \ell$; then $\mu\left(A_{n}\right) \rightarrow 0$.

If $\mathcal{L}$ is a lattice of subsets of $X$, then we will denote by:

$$
\begin{aligned}
& M_{\sigma}(\ell)=\text { the set of } \sigma \text {-smooth measures on } \mathcal{\ell} \text { of } M(\ell) \\
& M^{\sigma}(\mathcal{l})=\text { the set of } \sigma \text {-smooth measures on } \mathcal{\ell}(\ell) \text { of } M(\ell) \\
& M_{R}^{\sigma}(\ell)=\text { the set of } \ell \text {-regular measures of } M^{\sigma}(\ell)
\end{aligned}
$$

DEFINITION 2.3. If $A \in \mathcal{\Lambda}(\ell)$ and if $x \in X$ then $\mu_{x}(A)=\left\{\begin{array}{l}1 \text { if } x \in A \\ 0 \text { if } x \notin A\end{array}\right.$ is the measure concentrated at $x$.

$I(\ell)$ is the subset of $M(\ell)$ which consist of non-trivial zero-one valued measures.

$$
\begin{aligned}
& I_{R}(\ell)=\text { the set of } \ell \text {-regular measures of } I(\ell) \\
& I_{\sigma}(\ell)=\text { the set of } \sigma \text {-smooth measures on } \ell \text { of } I(\ell) \\
& I^{\sigma}(\ell)=\text { the set of } \sigma \text {-smooth measures on } \ell(\ell) \text { of } I(\ell) \\
& I_{R}^{\sigma}(\ell)=\text { the set of } \ell \text {-regular measures on } I^{\sigma}(\ell)
\end{aligned}
$$

DEFINITION 2.4. If $\mu \in M(\ell)$ then we define the support of $\mu$ to be:

$$
S(\mu)=\bigcap\{L \in \mathcal{L} \mid \mu(L)=\mu(X)\}
$$

Consequently if $\mu \in I(\ell)$

$$
S(\mu)=\bigcap\{L \in \mathcal{L} \mid \mu(L)=1\}
$$

DEFINITION 2.5. We say that the lattice $\ell$ is:

1) Replete if $S(\mu) \neq \emptyset$ for any $\mu \in I_{R}^{\sigma}(\ell)$.

2) Prime Complete if $S(\mu) \neq \emptyset$ for any $\mu \in I_{\sigma}(\ell)$ 
DEFINITION 2.6. Let $\pi: \ell \mapsto\{0,1\} ; \pi$ will be called a premeasure on $\ell$ if $\pi(X)=1, \pi$ is monotinic and multiplicative i.e., $\pi\left(L_{1} \cap L_{2}\right)=\pi\left(L_{1}\right) \cdot \pi\left(L_{2}\right)$ for $L_{1}, L_{2} \in \ell$. $\Pi(\ell)$ denotes all such premeasures defined on $\mathcal{L}$ and $\Pi_{\sigma}(\ell)$ represents $\sigma$-smooth premeasures on $\mathcal{L}$.

We now list a few well known facts which will enable us to characterize some previously defined properties in a measure theoretic fashion. The lattice $\mathcal{L}$ is:

1) Disjunctive if and only if $\mu_{x} \in I_{R}(\ell), \forall x \in X$.

2) $T_{2}$ if and only if $S(\mu)=0$ or a singleton for any $\mu \in I(\ell)$.

3) Compact if and only if $S(\mu) \neq 0$ for any $\mu \in I_{R}(\ell)$.

4) Countably compact if and only if $I_{R}(\ell)=I_{R}^{\sigma}(\ell)$

5) Lindelöf if and only if $S(\mu) \neq \emptyset$ for any $\mu \in \Pi_{\sigma}(\ell)$

6) Normal if and only if for any $\mu \in I(\ell)$ there exists a unique $\nu \in I_{R}(\ell)$ such that $\mu \leq \nu$ on $\ell$ FILTER AND MEASURE RELATIONSHIPS

Let $\mathcal{L}$ be a lattice of subsets of $X$.

DEFINITION 2.7 We say that $\mathscr{g} \subset \mathcal{L}$ is an $\mathcal{\ell}$-filter if:

(1) $\oslash \notin 9$

(2) If $L_{1}, L_{2} \in g \Rightarrow L_{1} \cap L_{2} \in \mathcal{F}$

(3) If $L_{1} \subset L_{2}$ and $L_{1} \in \mathscr{F} \Rightarrow L_{2} \in \mathcal{F}$

DEFINITION 2.8. $q$ is said to be a prime $\ell$-filter if:

(1) $I$ is an 2 -filter, and

(2) If $L_{1}, L_{2} \in \mathcal{L}$ and $L_{1} \cup L_{2} \in \mathscr{g} \Rightarrow L_{1} \in \mathcal{G}$ or $L_{2} \in \mathcal{G}$

DEFINITION 2.9. If $g$ is an $\ell$-filter we say that $g$ is an $\mathcal{\ell}$-ultrafilter if $g$ is a maximal $\ell$-filter. If $\mu \in I(\ell)$ let $\mathscr{F}_{\mu}=\{L \in \mathcal{L}: \mu(L)=1\}$.

PROPOSITION 2.10.

(1) If $\mu \in I(\ell)$, then $\mathscr{F}_{\mu}$ is an $\ell$-prime filter and conversely any $\ell$-prime filter determines an element $\mu \in I(\ell)$ and the correspondence is a bijection.

(2) If $\mu \in I_{R}(\ell)$, then $\sigma_{\mu}$ is an $\ell$-ultrafilter and conversely any $\ell$-ultrafilter determines an element $\mu \in I_{R}(\ell)$ this correspondence is also bijection. $\mathscr{T}_{\mu}$ is an $\ell$-ultrafilter if and only if $\mu \in I_{R}(\ell)$.

SEPARATION OF LATTICES

We are going to state a few known facts about the separation of lattices. We will use these results later on in the paper.

DEFINITION 2.11. Let $\ell_{1}$ and $\ell_{2}$ be two lattices of subsets of $X$. We say that $\ell_{1}$ separates $\ell_{2}$ if $A_{2}, B_{2} \in \mathcal{L}_{2}$ and $A_{2} \cap B_{2}=$ then there exists $A_{1}, B_{1} \in \mathcal{L}_{1}$ such that $A_{2} \subset A_{1}, B_{2} \subset B_{1}$ and $A_{1} \cap B_{1}=0$.

PROPOSITION 2.12. Let $\ell$ be a lattice of subset of $X . \quad \ell$ is compact if and only if $\tau \ell$ is compact, in which case $\ell$ separates $\tau \ell$

PROPOSITION 2.13. $\ell$ Lindelöf if and only if $\tau \ell$ is Lindelöf and in this case if $\ell$ is also $\delta$ then $\mathcal{L}$ separates $\tau \mathcal{L}$.

The proofs for these propositions are easy and will be omitted.

THEOREM 2.14. Suppose $\ell_{1} \subset \ell_{2}$ and $\ell_{1}$ separates $\ell_{2}$ then $\ell_{1}$ is normal if and only if $\ell_{2}$ is normal.

PROOF.

(1.) Suppose that $\ell_{1}$ is normal and let $A_{2}, B_{2} \in \ell_{2} ; A_{2} \cap B_{2}=0$. Since $\ell_{1}$ separates $\ell_{2}$ then there exist $A_{1}, B_{1} \in \ell_{1}$ such that $A_{2} \subset A_{1}, B_{2} \subset A_{2}$ and $A_{1} \cap B_{1}=0$. Now since $\ell_{1}$ is normal there exist $A, B \in \ell_{1} \subset \mathcal{L}_{2}$ such that $A_{1} \subset A^{\prime}, B_{1} \subset B^{\prime}$ and $A^{\prime} \cap B^{\prime}=0$. Therefore $A_{2} \subset A_{1} \subset A^{\prime}, B_{2} \subset B_{1} \subset B^{\prime}$ and $A^{\prime} \cap B^{\prime}=$ i.e., $\ell_{2}$ is normal. 
(2.) Suppose that $\ell_{2}$ is normal. Let $\mu_{1} \in I\left(\ell_{1}\right)$ and assume that there exist two measures $\nu_{1}, \tau_{1} \in I_{R}\left(\ell_{1}\right)$ and $\mu_{1} \leq \nu_{1}, \mu_{1} \leq \tau_{1}$ on $\ell_{1}$. Let $\mu_{2}, \nu_{2}$ and $\tau_{2}$ the respective extensions of the previous measures. Note that later two extensions are unique and belong to $I_{R}\left(\ell_{2}\right)$. Furthermore it can be seen since $\ell_{1}$ separates $\ell_{2}$ that $\mu_{2} \leq \nu_{2}$ and $\mu_{2} \leq \tau_{2}$ on $\ell_{2}$. However, since $\ell_{2}$ is normal then $\nu_{2}=\tau_{2}$ therefore $\nu_{1}=\tau_{1}$ and thence $\ell_{1}$ is normal.

\section{THE WALLMAN SPACE}

If $\mathcal{\ell}$ is a disjunctive lattice of subsets of an abstract set $X$ then there is a Wallman space associated with it. We will briefly review the fundamental properties of this Wallman space.

For any $A$ in $\Lambda(\ell)$, define $W(A)$ to be $W(A)=\left\{\mu \in I_{R}(\ell): \mu(A)=1\right\}$.

If $A, B \in \mathcal{l}(\ell)$ then:

1) $W(A \cup B)=W(A) \cup W(B)$.

2) $W(A \cap B)=W(A) \cap W(B)$.

3) $W\left(A^{\prime}\right)=W(A)^{\prime}$.

4) $W(A) \subset W(B)$ if and only if $A \subset B$.

5) $W(A)=W(B)$ if and only if $A=B$.

6) $W[\Lambda(\ell)]=\Lambda[W(\ell)]$.

Let $W(\ell)=\{W(L), L \in \ell\}$.

$W(\ell)$ is a compact lattice, and the topological space $I_{R}(\ell)$ with closed sets $\tau W(\ell)$ is a compact $T_{1}$ space called the Wallman space associated with $X$ and $\ell$. Since $\ell$ is disjunctive, it will be $T_{2}$ if and only if $\mathcal{L}$ is normal.

In addition to each $\mu \in M(\ell)$ there correspondence a unique $\hat{\mu} \in M(W(\ell))$, where $\widehat{\mu}(W(A))=\mu(A)$ for $A \in \mathcal{L}(\ell)$ and conversely. Also, $\mu \in M_{R}(\ell)$ if and only if $\hat{\mu} \in M_{R}(W(\ell))$. Since $W(\ell)$ is compact so is $\tau W(\ell)$, and $W(\ell)$ separates $\tau W(\ell)$ (see Proposition 2.11). Furthermore $\widehat{\mu} \in M_{R}(W(\ell)$ ) has a unique extension to $\tilde{\mu} \in M_{R}(\tau W(\ell))$.

Next we consider the space $I_{R}^{\sigma}(\ell)$ and its topology.

DEFINITION 2.15. Let $\ell$ be a disjunctive lattice of subsets of $X, L \in \ell$ and $A \in \ell(\ell)$.

1) $W_{\sigma}(L)=\left\{\mu \in I_{R}^{\sigma}(\ell) \mid \mu(L)=1\right\}$.

2) $W_{\sigma}(A)=\left\{\mu \in I_{R}^{\sigma}(\ell) \mid \mu(A)=1\right\}$.

3) $W_{\sigma}(\ell)=\left\{W_{\sigma}(L), L \in \ell\right\}=W(\ell) \cap I_{R}^{\sigma}(\ell)$.

The following properties hold and are not difficult to prove.

PROPOSITION 2.16. Let $\ell$ be a disjunctive lattice then for $A, B \in \mathcal{\ell}(\ell)$

1) $W_{\sigma}(A \cup B)=W_{\sigma}(A) \cup W_{\sigma}(B)$.

2) $W_{\sigma}(A \cap B)=W_{\sigma}(A) \cap W_{\sigma}(B)$.

3) $W_{\sigma}\left(A^{\prime}\right)=W_{\sigma}(A)^{\prime}$.

4) $W_{\sigma}(A) \subset W_{\sigma}(B)$ if and only if $A \subset B$.

5) $\Lambda\left[W_{\sigma}(\ell)\right]=W_{\sigma}[\Lambda(\ell)]$.

6) $\sigma\left[W_{\sigma}(l)\right]=W_{\sigma}[\sigma(l)]$.

For each $\mu \in M(\ell)$ there corresponds a unique $\mu^{\prime} \in M\left(W_{\sigma}(\ell)\right)$, where $\mu^{\prime}\left(W_{\sigma}(A)\right)=\mu(A)$ for $A \in \mathcal{L}(\ell)$ and conversely.

$\mu \in M_{R}(\ell)$ if and only if $\mu^{\prime} \in M_{R}\left(W_{\sigma}(\ell)\right)$, and

$\mu \in M_{\sigma}(\ell)$ if and only if $\mu^{\prime} \in M_{\sigma}\left(W_{\sigma}(\ell)\right)$

It can be shown that the lattice $W_{\sigma}(\ell)$ is replete and hat $I_{R}^{\sigma}(\ell)$ with $\tau W_{\sigma}(\ell)$ as the topology of closed sets is disjunctive and $T_{1}$. It will be $T_{2}$ if we further assume that property (P1) is satisfied; where $(\mathrm{P} 1)$ is defined as follows: 
(P1): For each $\mu \in I(\ell)$ there exists at most one $\nu \in I_{R}^{\sigma}(\ell)$ such that $\mu \leq \nu$ on $\ell$.

A proof of the last statement can be found in [8].

3. NORMAL AND STRONGLY NORMAL LATTICES

PROPOSITION 3.1. $\ell$ is normal if and only if for each $L \in \mathcal{L}$ where $L \subset L_{1}^{\prime} \cup L_{2}^{\prime}$ and $L_{1}, L_{2} \in \ell$; then there exists $A_{1}, A_{2} \in \mathcal{L}$ such that $A_{1} \subset L_{1}^{\prime}$ and $A_{2} \subset L_{2}^{\prime}$ and $L=A_{1} \cup A_{2}$.

PROOF.

(1.) Assume that $\mathcal{L}$ is normal and that $L \subset L_{1}^{\prime} \cup L_{2}^{\prime}$ then $L \cap L_{1} \cap L_{2}=\emptyset$ or equivalently $\left(L \cap L_{1}\right) \cap\left(L \cap L_{2}\right)=0$. Since $\mathcal{L}$ is normal there exist $\tilde{A}_{1}, \tilde{A}_{2} \in \mathcal{L}$ such that $L \cap L_{1} \subset \tilde{A}_{1}^{\prime}, L \cap L_{2} \subset \tilde{A}_{2}^{\prime}$ and $\tilde{A}_{1}^{\prime} \cap \tilde{A}_{2}=0$. Let $A_{1}=L \cap \tilde{A}_{1} \quad$ and $A_{2}=L \cap \tilde{A}_{2}$. Clearly $A_{1} \subset L_{1}^{\prime}$ and $A_{2} \subset L_{2}^{\prime}$. Now $A_{1} \cup A_{2}=\left(L L_{1} \cap \tilde{A}_{1}\right) \cup\left(L_{2} \cap \tilde{A}_{2}\right)=L \cap\left(\tilde{A}_{1} \cup \tilde{A}_{2}\right)=L \cap X=L$.

(2.) Let $L_{1} \cap L_{2}=$ and $L_{1}, L_{2} \in \mathcal{L}$ then $X=L_{1}^{\prime} \cup L_{2}^{\prime}$ and by the condition there exist $A_{1}, A_{2} \in \mathcal{L}$ such that $A_{1} \subset L_{1}^{\prime}, A_{2} \subset L_{2}^{\prime}$ and $A_{1} \cup A_{2}=X$, clearly $L_{1} \subset A_{1}^{\prime}, L_{2} \subset A_{2}^{\prime}$ and $A_{1}^{\prime} \cap A_{2}^{\prime}=\emptyset$ and thence $\mathcal{L}$ is normal.

DEFINITION 3.2. Let $\pi: \mathcal{L} \mapsto\{0,1\}$; $\pi$ will be called a premeasure on $\ell$ if $\pi(X)=1, \pi$ is monotonic and multiplicative i.e., $\pi\left(L_{1} \cap L_{2}\right)=\pi\left(L_{1}\right) \cdot \pi\left(L_{2}\right)$ for $L_{1}, L_{2} \in \ell$. $\Pi(\ell)$ denotes all such premeasures defined on $\ell$. It can be shown that there is a one-to-one correspondence between elements of $\Pi(\ell)$ and $\ell$-filters.

DEFINITION 3.3. Let $\tilde{I}(\ell)=\left\{\pi \in \Pi(\ell)\right.$ : if $L_{1} \cup L_{2}=X$ then $\pi\left(L_{1}\right)=1$ or $\left.\pi\left(L_{2}\right)=1\right\}$

Clearly, $\quad I_{R}(\ell) \subset I(\ell) \subset \tilde{I}(\ell) \subset \Pi(\ell)$

Let $\mathscr{T}=\{L \in \mathcal{L}: L \cap A \neq \emptyset$ for all $A \in \mathcal{L}$ such that $\pi(A)=1, \pi \in \tilde{I}(\ell)\}$

THEOREM 3.4. $\mathcal{L}$ is normal if and only if $\mathcal{G}$ is an $\ell$-ultrafilter.

PROOF.

(1.) Assume that $\mathcal{L}$ is normal we have to show that:

(a) $\emptyset \notin \mathcal{T}$ obvious

(b) If $L_{1} \subset L_{2}, L_{1} \in \mathcal{G} \Rightarrow L_{2} \in \mathcal{G}$

(c) If $L_{1}, L_{2} \in \mathcal{G} \Rightarrow L_{1} \cap L_{2} \in \mathcal{G}$

We have to show that $L_{1} \cap L_{2} \cap A \neq \emptyset$ for all $A \in \mathcal{L}$ such that $\pi(A)=1$. Assume otherwise i.e., $L_{1} \cap L_{2} \cap A=0$ for some $A \in \mathcal{L}$ and $\pi(A)=1$ where $\pi \in \tilde{I}(\mathcal{L})$ then $\left(L_{1} \cap A\right) \cap\left(L_{2} \cap A\right)=0$. Since $\mathcal{L}$ is normal there exist $A_{1}, A_{2} \in \mathcal{L}$ such that $L_{1} \cap A \subset A_{1}^{\prime}, L_{2} \cap A \subset A_{2}^{\prime}$ and $A_{1}^{\prime} \cap A_{2}^{\prime}=0$.

Clearly $A_{1} \cup A_{2}=X \Rightarrow \pi\left(A_{1} \cup A_{2}\right)=1 \Rightarrow \pi\left(A_{1}\right)=1$ or $\pi\left(A_{2}\right)=1$. Say $\pi\left(A_{1}\right)=1$. then $\pi\left(A \cap A_{1}\right)=1$ and $L_{1} \cap A_{1} \cap A=\emptyset$ which is a contradiction since $L_{1} \in \mathcal{G}$

(d) Now assume that $\mathscr{\sigma} \subset g$ where $g$ is an $\ell$-ultrafilter. Assume their exists $L \in g$ but $L \notin \mathcal{G}$, hence there exists $A \in \mathcal{L}$ such that $\pi(A)=1$ but $A \cap L=0$. However since $\pi(A)=1$ then $L \cap A \neq \emptyset$ for all

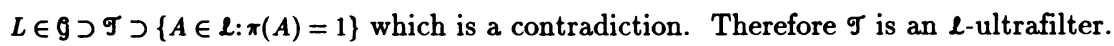

(2.) Now assume that $\mathcal{T}$ is an $\ell$-ultrafilter we have to show that $\ell$ is normal i.e., if $\mu \in I(\ell)$ there exists a unique $\nu \in I_{R}(\ell)$ such that $\mu \leq \nu$ on $\ell$. Suppose there exist $\nu_{1}, \nu_{2} \in I_{R}(\ell)$ and $\mu \leq \nu_{1} \mu \leq \nu_{2}$ on $\mathcal{L}$. Let $\mathscr{F}_{\mu}=\{L \in \mathcal{L}: \mu(L)=1\}$ and $\mathcal{\sigma}_{\mu}=\{L \in \mathcal{L}: L \cap A \neq \emptyset$ for all $A \in \mathcal{L}$ such that $\mu(A)=1\}$.

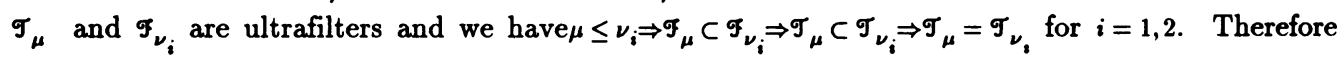
$\mathscr{\sigma}_{\mu}=\mathscr{T}_{\nu_{1}}=\mathscr{T}_{\nu_{2}}$. Furthermore we have that $\mathscr{F}_{\nu_{i}} \subset \mathscr{T}_{\nu_{i}}$ and hence $\mathscr{T}_{\mu}=\mathscr{T}_{\nu_{1}}=\mathscr{F}_{\nu_{2}}$.

Finally since all the ultrafilters are equal we get that $\nu_{1}=\nu_{2}$ which proves that $\ell$ is normal.

Let $\mu \in I(\ell)$. Define for any $E \subset X, \bar{\mu}(E)=$ inf,$\mu\left(L^{\prime}\right)$. Then it is easily seen that $\bar{\mu}$ is a finitely subadditive outer measure. $\underset{L \in \mathcal{L}}{E \subset L^{\prime}}$

PROPOSITION 3.5. $\mathcal{L}$ is normal if and only if $96=\{L \in \ell: \bar{\mu}(L)=1\}$ is a prime $\ell$-filter.

PROOF. Suppose $\mathcal{\ell}$ is normal. If $L_{1}, L_{2} \in 96$ then $\bar{\mu}\left(L_{1}\right)=\bar{\mu}\left(L_{2}\right)=1$. Now if $\bar{\mu}\left(L_{1} \cap L_{2}\right)=0$ then 
there exists $A \in \mathcal{L}$ such that $L_{1} \cap L_{2} \subset A^{\prime}$ and $\mu\left(A^{\prime}\right)=0$. But then $L_{1} \cap L_{2} \subset A^{\prime}$, and since $\mathcal{L}$ is normal, by Proposition 3.1, we have $A=A_{1} \cup A_{2}$ where $A_{1}, A_{2} \in \mathcal{L}, A_{1} \subset L_{1}^{\prime}$, and $A_{2} \subset L_{2}^{\prime}$. Now $\mu(A)=1$ then $\mu\left(A_{1}\right)=1$ or $\mu\left(A_{2}\right)=1$. Say $\mu\left(A_{1}\right)$, then $\mu\left(A_{1}^{\prime}\right)=0$ which is a contradiction since $\ell_{1} \subset A_{1}^{\prime}$ and $\bar{\mu}\left(L_{1}\right)=1$. Thus $\ell_{1}, L_{2} \in 96$ implies $L_{1} \cap L_{2} \in 96$.

The rest of the proof is clear.

THEOREM 3.6. Let $\pi \in \Pi(\ell)$ then:

$\pi \in \tilde{I}(\ell)$ if and only if there exists $\nu \in I(\ell)$ such that $\nu \leq \pi$.

PROOF. Suppose $x \in \tilde{I}(L)$ and let $\mathcal{A}=\left\{L^{\prime} \in^{\ell} \ell^{\prime}: \pi(L)=0\right\} . \quad \notin \mathbb{A}$ and $\mathcal{H}$ has the finite intersection property. The intersection of elements of $A$ form an $\ell^{\prime}$-filter base. Now assume that $\mathcal{M} \subset g$ and $g$ is $\ell^{\prime}$-ultrafilter. Then $g \leftrightarrow \rho \in I_{R}\left(\ell^{\prime}\right)$. If $\pi(L)=0$ then $L^{\prime} \in \mathcal{A} \subset g \Rightarrow \rho\left(L^{\prime}\right)=1 \Rightarrow \rho(L)=0$ hence $\rho \leq \pi$ on $\ell$ and therefore $\exists \nu \in I(\ell)$ such that $\nu=\rho \leq \pi$ on $\ell$.

The second part of the proof is easy and shall be omitted.

Let $36 \subset \ell, X \notin 36$ and if $L_{1}, L_{2} \in J_{6}$ then $L_{1} \cup L_{2} \in 36$. Consider the set of all $\ell$-filters $g_{\alpha}$ that exclude $\Im 6$, (i.e., $g_{\alpha} \cap \Im 6=\theta$ ). We partially order $g$ by set inclusion. Since $\{X\}$ is an $\ell$-filter that exclude 36 , then there exists at least one $g_{\alpha}$. Furthermore, since $\left\{\vartheta_{\alpha}, \subseteq\right\}$ is a partial ordering, which is an inductive ordering then by Zorn's lemma there must exist a maximal element. Let $\theta$ be this maximal element. So $g=\max \left\{\oint_{\alpha}\right.$ : where $\oint_{\alpha}$ are $\ell$-filters that exclude 36$\}$ and $g \neq 0$.

THEOREM 3.7. $g$ is a prime $\ell$-filter.

PROOF. $g$ is certainly an $\ell$-filter.

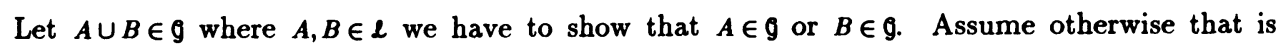
$A, B \notin g$. Suppose that $\exists F_{0} \in g$ such that $A \cap F_{0}=$ then $F_{0} \cap(A \cup B) \in g \Rightarrow\left(F_{0} \cap A\right) \cup\left(F_{0} \cap B\right)$ $=F_{0} \cap B \in g \Rightarrow B \in Q$ which is a contradiction thus we may now assume that $A \cap F \neq \emptyset$ and $B \cap F \neq 0$ for all $F \in g$.

Let $\sigma_{1}$ be the filter generated by all $\{A \cap F \mid F \in g\}$. Since $A \in \mathcal{F}_{1}$ and $A \notin g \Rightarrow g \subset \mathcal{\sigma}_{1}$, similarly let

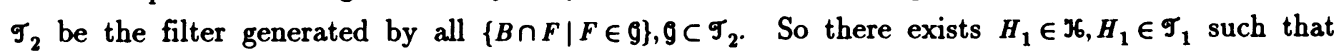
$A \cap F_{1} \subset H_{1}$ for some $F_{1} \in Q$ and similarly there exists $H_{2} \in W_{6} H_{2} \in \mathcal{F}_{2}$ such that $A \cap F_{2} \subset H_{2}$ for some $F_{2} \in g$. Let $F_{1} \cap F_{2}=F_{3}$ then $H_{1} \cup H_{2} \supset\left(A \cap F_{1}\right) \cup\left(B \cap F_{2}\right) \supset\left(A \cap F_{3}\right) \cup\left(B \cap F_{3}\right) \Rightarrow H_{1} \cup H_{2} \supset(A \cup B)$ $\cap F_{3} \in g$ however since $H_{1} \cup H_{2} \in \Im$, it is a contradiction. Thus $A \in g$ or $B \in \mathcal{G}$ or equivalently $g$ is a prime filter; and so $g \leftrightarrow \mu \in I(\ell)$.

COROLLARY 3.8. Let $\pi \in \Pi(l)$ then $\pi=\bigwedge_{\pi<\mu_{\alpha}} \mu_{\alpha}$

$$
\begin{aligned}
\pi<\mu_{\alpha} \\
\mu_{\alpha} \in I(\mathcal{L})
\end{aligned}
$$

PROOF. Let $\mathscr{g}$ be the $\ell$-filter representing $\pi$ i.e., $\mathscr{F}=\{L \in \mathcal{L}: \pi(L)=1\}$. Let $g_{\alpha}$ be the prime $\ell$ filter representing $\mu_{\alpha}$, so; $g_{\alpha}=\left\{L \in \mathcal{L}: \mu_{\alpha}(L)=1\right\}$. Clearly $\mathscr{G} \subseteq \bigcap_{\mathcal{G}_{\subset} \mathfrak{g}_{\alpha}} g_{\alpha \cdot}$ We have to show that $\mathscr{f} \supseteq \bigcap_{\mathcal{F} \subset g_{\alpha}} g_{\alpha}$.

Assume that there exists $A \in \mathcal{L}$ and $A \in g_{\alpha}$ for all $\alpha$ but $A \notin 9$. Let $36=A$ then $A \neq X$ i.e., $X \notin 36$. Let 9 be a maximal $\ell$-filter containing 9 and excluding 36 . From the previous theorem, $g$ is a prime $\ell$ filter and $A \notin Q$, which is a contradiction; since $A$ belongs to all prime $\ell$-filters that contain 9 . Therefore $g=\bigcap_{\mathcal{F} \subset g_{\alpha}} g_{\alpha}$, and hence $\pi \in \Pi(\ell)$. Thus $\pi=\bigwedge_{\substack{x \leq \mu_{\alpha} \\ \mu_{\alpha} \in I(\ell)}} \mu_{\alpha}$

DEFINITION 3.9. We say that $\ell$ is strongly normal if for $\mu, \mu_{1}, \mu_{2} \in I(\ell)$ and $\mu \leq \mu_{1}, \mu \leq \mu_{2}$ on $\ell$; then $\mu_{1} \leq \mu_{2}$ or $\mu_{2} \leq \mu_{1}$ on $\ell$.

THEOREM 3.10. $\ell$ is strongly normal if and only if $I(\ell)=\tilde{I}(\ell)$ 


\section{PROOF.}

(1.) Suppose that $I(\ell)=\tilde{I}(\ell)$. Let $\mu_{1}, \mu_{2} \in I(\ell)$ and suppose that they are not comparable i.e., $\mu_{1} \leftleftarrows \mu_{2}$ and $\mu_{2} \leftleftarrows \mu_{1}$ on $\ell$. Then $\exists L_{1}, L_{2} \in \mathcal{L}$ such that $\mu_{2}\left(L_{j}\right)=\delta_{1}$. Consider $\mu_{1} \wedge \mu_{2}$. We have $\left(\mu_{1} \wedge \mu_{2}\right)\left(L_{1} \cup L_{2}\right)=1$ but $\left(\mu_{1} \wedge \mu_{2}\right)\left(L_{1}\right)=0$ and $\left(\mu_{1} \wedge \mu_{2}\right)\left(L_{2}\right)=0$ therefore $\mu_{1} \wedge \mu_{2} \notin I(\ell)$.

Now suppose that $\pi \in \tilde{I}(\ell)$, and $\pi \leq \mu_{1}$ and $\pi \leq \mu_{2}$ on $\ell$, then $\pi \leq \mu_{1} \wedge \mu_{2}$. Suppose $L_{1} \cup L_{2}=X$ then $\pi\left(L_{1}\right)=1$ or $\pi\left(L_{2}\right)=1$ say $\pi\left(L_{1}\right)=1 \Rightarrow\left(\mu_{1} \wedge \mu_{2}\right)\left(L_{1}\right)=1$ therefore $\mu_{1} \wedge \mu_{2} \in \tilde{I}(\ell)=I(\ell)$ which is a contradiction unless $\mu_{1} \leq \mu_{2}$ or $\mu_{2} \leq \mu_{1}$ on $\ell$. Therefore $\tilde{I}(\ell)=I(\ell) \Rightarrow \ell$ strongly normal.

(2.) Conversely assume $\ell$ is strongly normal. Let $\pi \in \tilde{I}(\ell)$ then $\pi=\bigwedge\left\{\mu_{\alpha}: \pi \leq \mu_{\alpha}, \mu_{\alpha} \in I(\ell)\right\}$. $\left\{\left\{\mu_{\alpha}\right\}_{\alpha \in \Lambda}, \leq\right\}$ is totally ordered. So $\mu_{\alpha} \leq \mu_{\beta}$ or $\mu_{\beta} \leq \mu_{\alpha}, \forall \alpha, \beta \in \Lambda$. Suppose $L_{1}, L_{2} \in \mathcal{L}$ and $\pi\left(L_{1} \cup L_{2}\right)=1$ then $\mu_{\alpha}\left(L_{1} \cup L_{2}\right)=1$ for all $\alpha$. Suppose that for some $\alpha_{0}, \mu_{\alpha_{0}}\left(L_{1}\right)=0$ then $\mu_{\gamma}\left(L_{1}\right)=0, \mu_{\gamma}\left(L_{2}\right)=1$ for all $\mu_{\gamma} \leq \mu_{\alpha_{0}}$ but then $\mu_{\beta}\left(L_{2}\right)=1$ for all $\mu_{\beta} \geq \mu_{\alpha_{0}}$. Hence $\mu_{\alpha}\left(L_{2}\right)=1$ for all $\alpha$, then $\pi\left(L_{2}\right)=1 \Rightarrow \pi \in I(\ell)$. Therefore $\tilde{I}(\ell)=I(\ell)$ if $\ell$ is strongly normal.

4. SOME PROPERTIES $W_{\sigma}(\ell)$.

We now consider the topological space $\left(I_{R}^{\sigma}(\ell), \tau W_{\sigma}(\ell)\right)$. Let (P2) be the following property.

(P2): If $\mu \in I_{\sigma}(\ell)$ then there exists $\nu \in I_{R}^{\sigma}(\ell)$ such that $\mu \leq \nu$ on $\ell$.

THEOREM 4.1. Let $\ell$ be a disjunctive lattice then $W_{\sigma}(\ell)$ is prime complete if and only if (P2) holds.

\section{PROOF.}

(1.) Suppose that $W_{\sigma}(\ell)$ is prime complete. Let $\mu \in I_{\sigma}(\ell)$ then $\mu^{\prime} \in I_{\sigma}\left[W_{\sigma}(\ell)\right]$ and since $W_{\sigma}(\ell)$ is prime complete then $S\left(\mu^{\prime}\right) \neq \emptyset$, however $S\left(\mu^{\prime}\right)=\left\{\nu \in I_{R}^{\sigma}(\ell) \mid \mu \leq \nu\right.$ on $\left.\ell\right\}$ then $\exists \nu \in I_{R}^{\sigma}(\ell)$ such that $\mu \leq \nu$ on $\mathcal{L}$ i.e., that (P2) is satisfied.

(2.) Suppose (P2) holds. Let $\lambda \in I_{\sigma}\left[W_{\sigma}(\ell)\right]$ then $\exists \mu \in I_{\sigma}(\ell)$ such that $\lambda=\mu^{\prime} \in I_{\sigma}\left[W_{\sigma}(\ell)\right]$. From (P2) $\exists \nu I_{R}^{\sigma}(\ell) \mid \mu \leq \nu$ on $\ell$. Hence $\mu^{\prime} \leq \nu^{\prime}$ on $W_{\sigma}(\ell)$ where $\nu^{\prime} \in I_{r}^{\sigma}\left[W_{\sigma}(\ell)\right]$. Since $W_{\sigma}(\ell)$ is replete then $S\left(\nu^{\prime}\right) \neq \emptyset$, and $S\left(\nu^{\prime}\right) \subset S\left(\mu^{\prime}\right)=S(\lambda)$ then $S(\lambda) \neq 0$.

Let (P3) be the following property.

(P3): If $\pi \in \Pi_{\sigma}(\ell)$ there exists $\mu \in I_{R}^{\sigma}(\ell)$ such that $\pi \leq \mu$ on $\ell$.

\section{THEOREM 4.2.}

(1) If $\ell$ is replete and satisfies (P3) $\Rightarrow \ell$ is Lindelöf

(2) If $\ell$ is countably compact $\Rightarrow \ell$ satisfies (P3)

(3) If $\ell$ is disjunctive and Lindelöf $\Rightarrow \ell$ satisfies (P3)

(4) If $\mathcal{\ell}$ is disjunctive then, $\ell$ satisfies (P3) if and only if $\left(I_{R}^{\sigma}(\ell), \tau W_{\sigma}(\ell)\right)$ is Lindelöf

PROOF.

(1.) Let $\pi \in \Pi_{\sigma}(\ell)$ since $\ell$ satisfies (P3) $\exists \mu \in I_{R}^{\sigma}(\ell) \mid \pi \leq \mu$ on $\mathcal{L}, S(\mu) \neq \emptyset$ because $\ell$ is replete and $S(\mu) \subset S(\pi)$. Hence $S(\pi) \neq 0$.

(2.) Let $\pi \in \Pi_{\sigma}(\ell) \exists, \mu \in I_{R}(\ell) \mid \pi \leq \mu$. Since $\mathcal{\ell}$ is countably compact then $I_{R}(\ell)=I_{R}^{\sigma}(\mathcal{L})$. Hence $\ell$ satisfies (P3).

(3.) Let $\pi \in \Pi_{\sigma}(\mathcal{l})$ then $S(\pi) \neq \emptyset$ because $\mathcal{L}$ is Lindelöf. Let $x \in S(\pi) \Rightarrow \pi \leq \mu_{x} \in I_{R}^{\sigma}(\mathcal{l})$.

(4.) Assume $\ell$ satisfies (P3) then $W_{\sigma}(\ell)$ satisfies (P3) plus $W_{\sigma}(\ell)$ is always replete then $W_{\sigma}(\ell)$ is Lindelöf from part 1.

Conversely if $\left(I_{R}^{\sigma}(\ell), \tau W_{\sigma}(\ell)\right)$ is Lindelöf then $W_{\sigma}(\ell)$ is disjunctive and Lindelöf then $W_{\sigma}(\ell)$ satisfies (P3) from part 3 and hence $\ell$ satisfies (P3).

Define $V_{\sigma}(\ell)=\left\{\mu \in I_{\sigma}(\ell) \mid \mu(L)=1\right\}$ and $V_{\sigma}(\ell)=\left\{V_{\sigma}(L) \mid L \in \ell\right\}$. Similarly we can consider the set $I_{\sigma}(\ell)$ and the topology of closed set on $I_{\sigma}(\ell)$ given by $\tau V_{\sigma}(\ell)$.

Let (P4) be the following property. 
(P4): If $\pi \in \Pi_{\sigma}(\ell)$ then there exists $\mu \in I_{\sigma}(\ell)$ such that $\pi \leq \mu$ on $\ell$.

THEOREM 4.3.

1) If $\mathcal{L}$ is prime complete and satisfies $(\mathrm{P} 4) \Rightarrow \ell$ is Lindelöf

2) If $\ell$ is countably compact $\Rightarrow \ell$ satisfies (P4)

3) If $\ell$ is Lindelöf $\Rightarrow \ell$ satisfies (P4)

4) $\ell$ satisfies (P4) if and only if $\left(I_{R}^{\sigma}(\ell), \tau V_{\sigma}(\ell)\right.$ is Lindelöf

PROOF. The proof is similar to that of Theorem 4.2 and will be omitted.

REMARK. Consider once more the topological space $\left(I_{R}^{\sigma}(\ell), \tau W_{\sigma}(\ell)\right)$, where as usual we assume that $\ell$ is disjunctive. If $\ell$ is normal and if $W_{\sigma}(\ell)$ separates $r W_{\sigma}(\mathcal{L})$ then using (Theorem 2.13), we have that the topological space $\left(I_{R}^{\sigma}(\ell), \tau W_{\sigma}(\ell)\right)$ is normal. Finally, we note that if $\ell$ is a $\delta$ lattice then so is $W_{\sigma}(\ell)$ and therefore if $W_{\sigma}(\ell)$ is Lindelöf, then by (Theorem 2.12), $\tau W_{\sigma}(\ell)$ is Lindelöf and $W_{\sigma}(\ell)$ separates $\tau W_{\sigma}(\ell)$.

ACKNOWLEDGEMENT. The author wishes to express his appreciation to the referee for the many helpful and constructive comments which aided in the writing of the paper.

\section{REFERENCES}

1. ALEXANDROV, A.D., Additive set functions in abstract spaces, Mat. Sb. N.S. 2 (1937), 947972.

2. BACHMAN, G. \& STRATIGOS, P., On general lattice repleteness and completeness, Illinois J. Math. 27, No. 6 (1983), 535-561.

3. CAMACHO, J., On maximal measures with respect to a lattice, Internat. J. Math. \& Math. Sci. 14, No. 1 (1991), 93-98.

4. EID, G., On normal lattices and Wallman spaces, Internat. J. Math. \& Math. Sci. 13, No. 1 (1990), 31-38.

5. FROLIK, Z., Prime filters with c.i.p., Comment. Math. Univ. Carolina 13, No. 3 (1972), 553573.

6. SZETO, M., Measure repleteness and mapping preservations, J. Ind. Math. Soc. 43 (1979), 3552.

7. WALLMAN, H., Lattices and topological spaces, Ann. Math. 39 (1938), 112-126.

8. YALLAOUI, E.B., Induced measures on Wallman spaces, Internat. J. Math. \& Math. Sci. 13, No. 4 (1990), 783-798. 


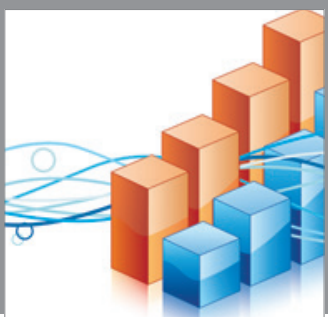

Advances in

Operations Research

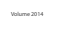

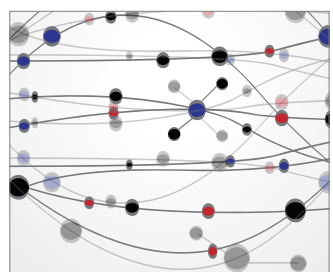

\section{The Scientific} World Journal
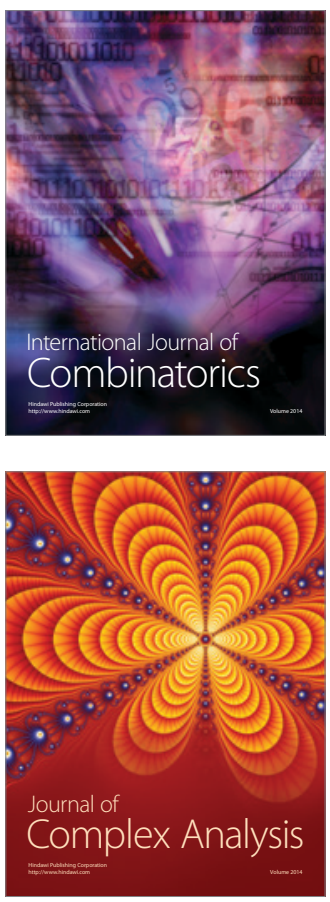

International Journal of

Mathematics and

Mathematical

Sciences
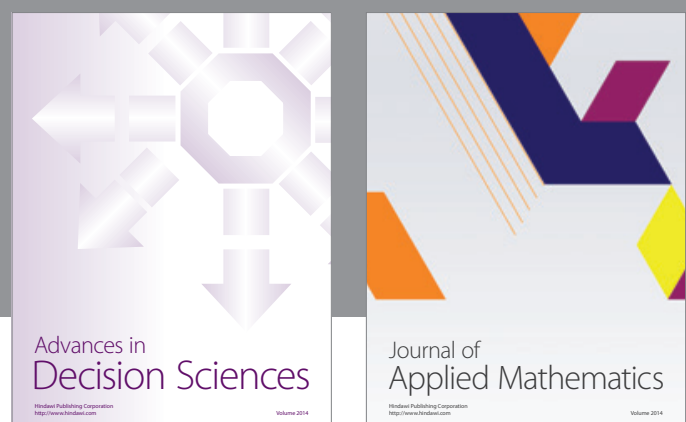

Journal of

Applied Mathematics
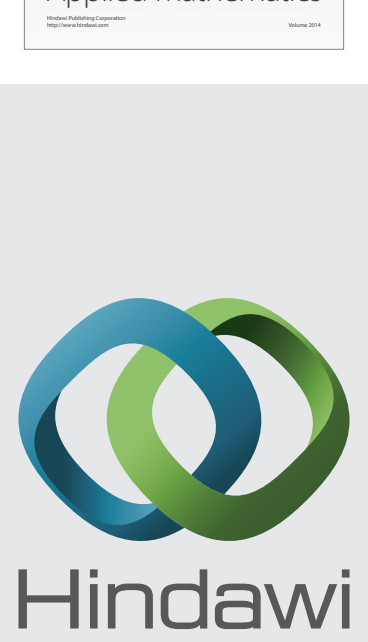

Submit your manuscripts at http://www.hindawi.com
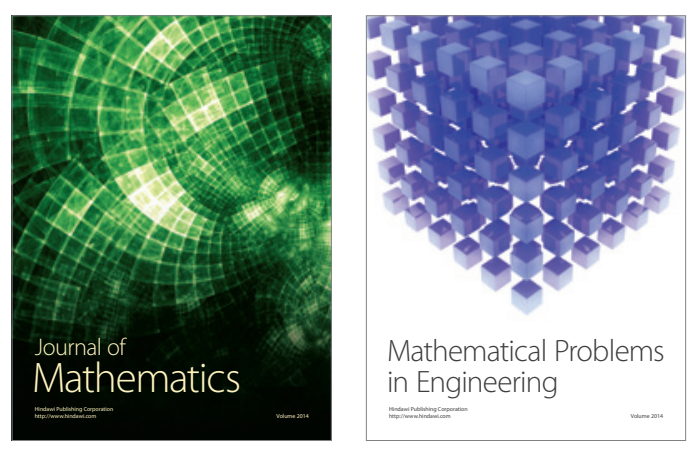

Mathematical Problems in Engineering
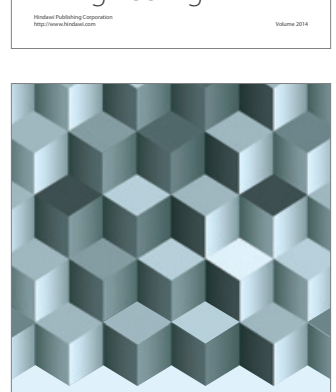

Journal of

Function Spaces
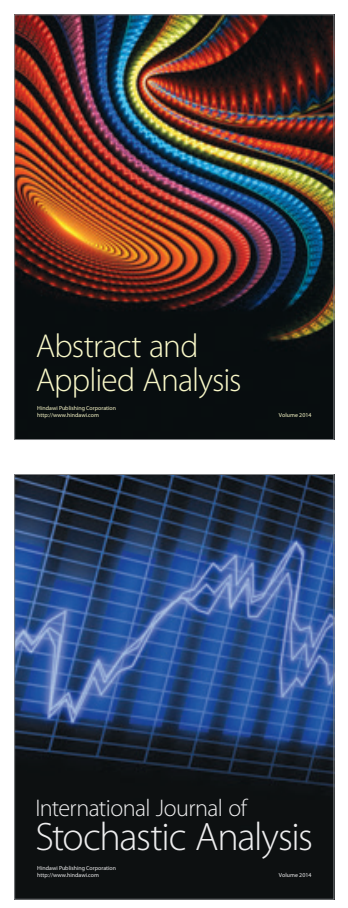

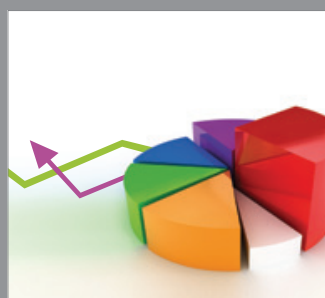

ournal of

Probability and Statistics

Promensencen
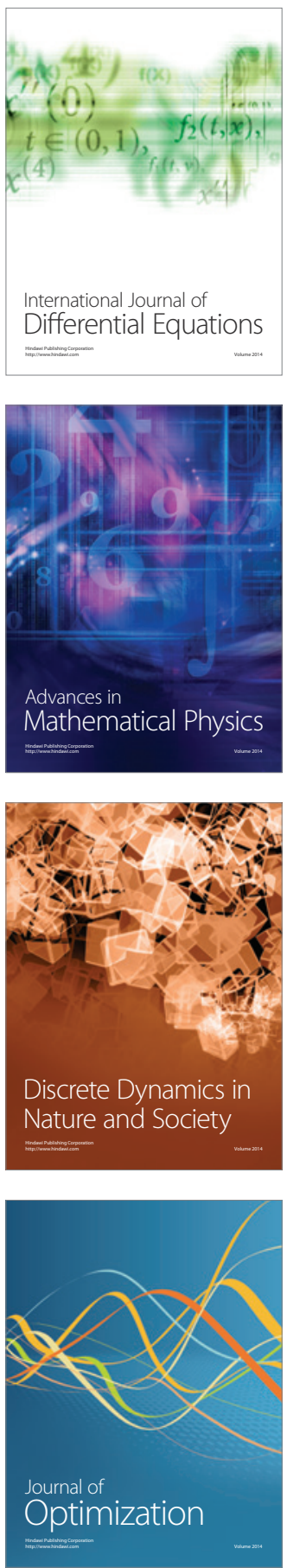